\title{
Aminochelin, a Catecholamine Siderophore Produced by Azotobacter vinelandii
}

\author{
By WILLIAM J. PAGE* AND MARGARET VON TIGERSTROM \\ Department of Microbiology, University of Alberta, Edmonton, Alberta T6G 2E9, Canada
}

(Received 9 July 1987; revised 6 October 1987)

\begin{abstract}
A catecholamine siderophore, named aminochelin, produced by iron-limited Azotobacter vinelandii was purified and tentatively identified as 2,3-dihydroxybenzoylputrescine. This compound was first observed as an ethyl-acetate-insoluble catechol that accounted for 30 to $50 \%$ of the total catechol in iron-limited culture supernatant fluids. The purified compound was unstable at neutral to alkaline $\mathrm{pH}$, bound $\mathrm{Fe}^{3+}, \mathrm{Fe}^{2+}$ and molybdate, and promoted ${ }^{55} \mathrm{Fe}$-uptake into iron-limited $A$. vinelandii. Aminochelin was induced and repressed coordinately with the other catechol siderophore azotochelin. The catechol siderophores were, however, less sensitive to repression by soluble iron than the yellow-green fluorescent peptide siderophore azotobactin.
\end{abstract}

\section{INTRODUCTION}

Optimal growth of Azotobacter vinelandii requires an adequate supply of iron. This iron is essential not only to support the obligate aerobic life of this bacterium, but also for nitrogen fixation and protection of the oxygen-labile nitrogenase enzyme (Post et al., 1983). Although iron is plentiful in soil, the iron minerals that form in aerobic soil layers at neutral pH are very insoluble. A. vinelandii, like other bacteria (Neilands, 1981), can accumulate the very low levels of soluble iron by using siderophore-mediated transport systems (Page \& Huyer, 1984; Knosp et al., 1984). In addition to the deployment of these high affinity chelators, $A$. vinelandii also produces 2,3-dihydroxybenzoic acid (2,3-DHBA), which solubilizes iron minerals and may facilitate low-affinity iron uptake in the absence of true siderophores (Page \& Huyer, 1984). This latter strategy appears to be common to the Azotobacteraceae as Azomonas macrocytogenes and Azotobacter paspali similarly use 3,4-dihydroxybenzoic acid (Westerveld et al., 1985; Collinson et al., 1987) and Azotobacter chroococcum employs citric acid to solubilize iron (Page, 1987).

When 2,3-DHBA is unable to provide sufficient iron for growth of $A$. vinelandii, the true siderophores are induced. This follows an unusual sequential derepression pattern, where the production of the catechol azotochelin [ $N, N^{1}$-bis(2,3-dihydroxybenzoyl)-L-lysine] precedes the production of the fluorescent yellow-green peptide azotobactin (Page \& Huyer, 1984; Page \& Grant, 1987). Certainly the nature of this sequential control is of interest as it will likely invoke additional levels of complexity on the model for iron-regulation of siderophore synthesis being developed in Escherichia coli (De Lorenzo et al., 1987; Hantke, 1981, 1984). Before regulation can be addressed, all of the major components of the $A$. vinelandii siderophore system should be known. In this study, we report that a second catechol siderophore, not previously described and different from azotochelin, is produced by iron-limited $A$. vinelandii.

\section{METHODS}

Bacterial strains and growth conditions. The capsule-negative strain UW of $A$. vinelandii $\mathrm{OP}$ and a spontaneous variant which failed to produce azotobactin (strain UAl) were used. These strains were maintained on Burk medium, $\mathrm{pH} 7 \cdot 2$, containing $1 \%(\mathrm{w} / \mathrm{v})$ glucose, $1 \cdot 1 \mathrm{~g}$ ammonium acetate $\mathrm{I}^{-1}$ and $1.8 \%(\mathrm{w} / \mathrm{v})$ agar, incubated at

Abbreviations: EA, ethyl acetate; 2,3-DHBA, 2,3-dihydroxybenzoic acid. 
$30^{\circ} \mathrm{C}$ as described previously (Page \& Huyer, 1984). Iron-limited Burk medium contained no added iron. The inoculum for each study was prepared from iron-starved cells (Page \& Huyer, 1984). Soluble iron was supplied as ferric citrate. Insoluble iron was provided as finely ground iron minerals or chemicals $(50 \mathrm{mg})$ added to $100 \mathrm{ml}$ iron-limited medium in a $500 \mathrm{ml}$ Erlenmeyer flask. Liquid medium was incubated at 28 to $30{ }^{\circ} \mathrm{C}$ with shaking at 225 r.p.m.

Siderophore analysis. After removal of cells by centrifugation, the culture supernatant fluid was acidified to pH 1.8 with $\mathrm{HCl}$ and scanned with a Perkin-Elmer Lambda 3 spectrophotometer. Absorbance at $310 \mathrm{~nm}$ was used to estimate total catechol and absorbance at $380 \mathrm{~nm}$ was used to estimate azotobactin (Page \& Huyer, 1984). Azotochelin plus 2,3-DHBA were extracted from acidified culture supernatant fluid as previously described (Corbin \& Bulen, 1969). Catechols were detected by the Arnow (1937) assay and hydroxamates were tested for by the Csaky (1948) assay.

Purification of aminochelin. Strain UW was grown in iron-limited Burk medium containing $50 \mathrm{mg}$ micaceous haematite per $100 \mathrm{ml}$ to enhance siderophore production (Page \& Huyer, 1984). After $20 \mathrm{~h}$ incubation, the cells were removed by centrifugation and the culture supernatant fluid was sterilized by filtration. After ethyl acetate (EA) extraction, the aqueous phase was neutralized and concentrated twofold by rotary evaporation at $28^{\circ} \mathrm{C}$, with protection from the light.

The aqueous phase containing EA-insoluble catechol (aminochelin, $A_{310}=62$ in $88 \mathrm{ml}$ ) was applied to a $2.6 \times 40 \mathrm{~cm}$ column containing DEAE Sephadex A25 (Pharmacia). The column had been previously washed with $500 \mathrm{ml} 3 \mathrm{~mm}$-Tris $/ \mathrm{HCl}$, pH 7.5, followed by $250 \mathrm{ml}$ distilled water. The sample was eluted with $100 \mathrm{ml}$ distilled water followed by an $800 \mathrm{ml}$ gradient formed with distilled water and $2 \mathrm{M}-\mathrm{NaCl}$. Elution of aminochelin was followed by measuring $A_{310}$.

Pooled fractions containing aminochelin were diluted with distilled water to give an $A_{310}=68.5$ in $50 \mathrm{ml}$ which was loaded onto a $2.6 \times 40 \mathrm{~cm}$ column of CM Sephadex (Pharmacia). The column had been equilibrated in $0.005 \mathrm{M}$-ammonium acetate, $\mathrm{pH} 6.8$. The sample was washed with $50 \mathrm{ml}$ of the same solution and then eluted with a $500 \mathrm{ml}$ gradient formed from $0.005 \mathrm{M}$ and $1.0 \mathrm{M}$-ammonium acetate.

Aminochelin fractions from this column were pooled and extracted with an equal volume of butanol. Aminochelin partitioned equally into butanol and water each time the extraction was repeated, so close to $100 \%$ recovery was possible after repeated extractions. The butanol fraction was reduced to dryness under an airstream and stored at $-20^{\circ} \mathrm{C}$. The dry material was a clear whitish residue, with only a trace of salts, and was completely soluble in water.

Thin layer chromatography $(T L C)$. Chromatography on silica gel $\mathrm{G}$ plates used the following solvent systems for catechol separations: (A) benzene/acetic acid/water (125:72:3, by vol.) and (B) butanol/acetic acid/water (150:30:30, by vol.). Amino compounds were separated using (C) $95 \%$ (v/v) ethanol/ammonium hydroxide/water (180:10:10, by vol.) and (D) propan-1-ol/ammonium hydroxide $(63: 33, \mathrm{w} / \mathrm{w})$.

Spray reagents included ninhydrin in acetone for amines, potassium ferricyanide-ferric chloride and $2,2^{\prime}$ dipyridyl-ferric chloride for phenols (Krebs et al., 1969) and the Arnow (1937) reagents for catechols.

Stability and hydrolysis of aminochelin. The stability of aminochelin was examined by spectrophotometric scanning and TLC after storage, for 4 and $20 \mathrm{~h}$, at $20^{\circ} \mathrm{C}$ and $4{ }^{\circ} \mathrm{C}$, in the dark and in the light, in the following: $5 \mathrm{~mm}-\mathrm{HCl}$, pH 2.5; distilled water, pH 5.2; 5 mM-ammonium acetate, pH 5.5; 25 mM-potassium phosphate, $\mathrm{pH} 7.0 ; 25 \mathrm{~mm}-\mathrm{Tris} / \mathrm{HCl}, \mathrm{pH} 7 \cdot 5$; and $5 \mathrm{~mm}-\mathrm{NaOH}, \mathrm{pH} 10.5$.

Acid hydrolysis of aminochelin required at least $6 \mathrm{M}-\mathrm{HCl}$ at $90-100^{\circ} \mathrm{C}$ for $25 \mathrm{~h}$. Free catechol was removed from the hydrolysate by ethyl ether extraction. This extract was concentrated by evaporation and dissolved in $95 \%(\mathrm{v} / \mathrm{v})$ ethanol before TLC analysis (Ong et al., 1979). The presence of 2,3-DHBA was qualitatively determined by comparison with authentic standards.

The masses of aminochelin and its hydrolysis products were determined by positive ion fast atom bombardment spectroscopy, using samples dissolved in glycerol.

${ }^{55} \mathrm{Fe}$-uptake measurement. The nitrogen-fixing strain $\mathrm{UW}$, pregrown for $16 \mathrm{~h}$ in iron-limited Burk medium (lacking ammonium acetate), was used for ${ }^{55} \mathrm{Fe}$-uptake measurement as described previously (Knosp et al., 1984). Purified azotochelin and azotobactin were also prepared as described previously (Knosp et al., 1984).

\section{RESULTS}

\section{Observation of a second catechol siderophore}

Spectrophotometric scans of acidified iron-limited $A$. vinelandii culture supernatant fluid revealed a peak at $380 \mathrm{~nm}$ which corresponded to azotobactin and a peak at $310 \mathrm{~nm}$ which corresponded to catechols (Fig..1). EA extraction of this acidified culture fluid removed the catechols azotochelin and 2,3-DHBA, but approximately $37 \%$ of the original $A_{310}$ material remained in the aqueous phase (Fig. 1). No further removal of the $A_{310}$ material occurred after a 


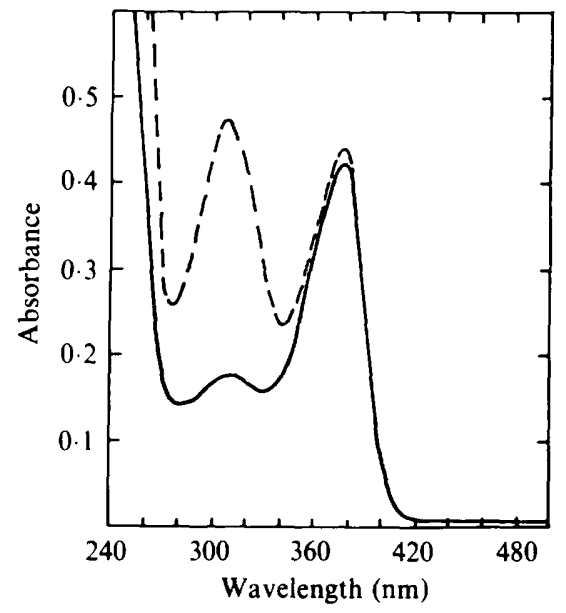

Fig. 1. Spectrophotometric scan of acidified iron-limited culture supernatant fluid before (-- $)$ and after $(\longrightarrow)$ EA extraction.

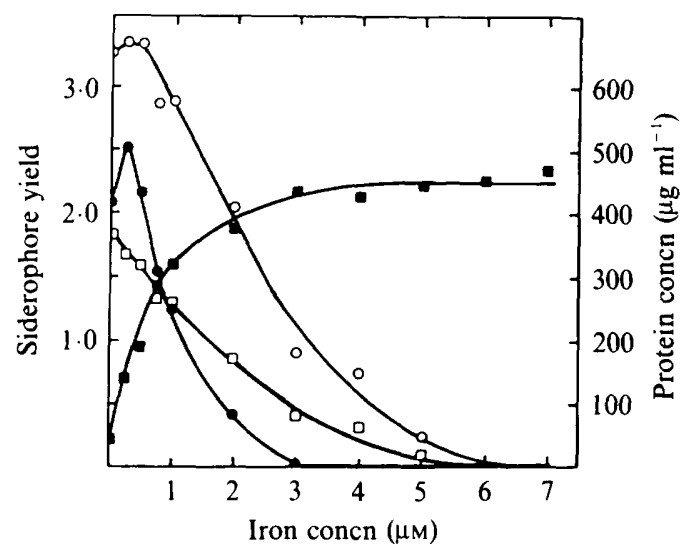

Fig. 2. Yields of azotobactin (O), total catechols before EA extraction $(O)$ and EA-insoluble catechol $(\square)$, after $22 \mathrm{~h}$ growth in medium containing various concentrations of ferric citrate. Siderophore yields were calculated as $A_{380}$ (azotobactin) or $A_{310}$ (catechols) (mg cell protein) ${ }^{-1}$. Cell yield is shown as $\mu \mathrm{g}$ protein $\mathrm{ml}^{-1}(\mathbf{G})$.

third extraction with EA. When an aqueous solution of azotochelin plus 2,3-DHBA was similarly extracted with EA, less than $5 \%$ of the $A_{310}$ material remained in the aqueous phase. Therefore, the remaining $A_{310}$ material was not simply residual azotochelin plus 2,3-DHBA that was in equilibrium with the EA-saturated water.

The EA-insoluble material was strongly Arnow positive, which indicated the presence of catechol, but was negative in the Csaky test for hydroxamate. Addition of $\mathrm{Fe}^{3+}$ to this material resulted in a purple colour, as would be expected from iron chelation by a catechol.

In iron-limited culture, azotochelin was first detected after $8 \mathrm{~h}$ incubation (Page \& Grant, 1987) which coincided with the first appearance of the EA-insoluble catechol (data not shown). The repression of the EA-insoluble catechol by iron also paralleled azotochelin repression and both were less tightly controlled by iron availability than azotobactin (Fig. 2). In iron-limited medium the EA-insoluble catechol accounted for approximately $50 \%$ of the total $A_{310}$ material and this decreased to a constant 38 to $44 \%$ of the total in cultures containing 1.0 to $5.0 \mu \mathrm{M}-\mathrm{Fe}^{3+}$. 


\section{Table 1. Presence of EA-insoluble catechol in cultures grown with mineral iron sources}

Minerals were added at $50 \mathrm{mg}$ per $100 \mathrm{ml}$ of iron-limited medium and incubated at $28^{\circ} \mathrm{C}$ with shaking at 225 r.p.m. for $22 \mathrm{~h}$. The absorbance values of acidified culture fluids and the percentage of total $A_{310}$ remaining in the aqueous phase after EA extraction are shown. Values shown are the means of duplicate experiments. Absorbance values were reproducible to \pm 10 to $15 \%$ of the mean. Values for percentage of $A_{310}$ insoluble in EA were reproducible to $\pm 2 \%$ of the mean.

\begin{tabular}{|c|c|c|c|c|c|c|}
\hline \multirow[b]{2}{*}{ Mineral } & \multirow[b]{2}{*}{ Ideal formula } & \multicolumn{3}{|c|}{ Strain UW } & \multicolumn{2}{|c|}{ Strain UAl } \\
\hline & & $A_{380}$ & $A_{310}$ & $\begin{array}{c}\text { Percentage } \\
A_{310} \text { insoluble } \\
\text { in EA }\end{array}$ & $A_{310}$ & $\begin{array}{l}\text { Percentage } \\
A_{310} \text { insoluble } \\
\text { in EA }\end{array}$ \\
\hline None & - & $0 \cdot 340$ & $0 \cdot 370$ & 53 & 0.410 & 41 \\
\hline Pyrrhotite & $\mathrm{Fe}_{1-x} \mathrm{~S}$ & 0.966 & 1.860 & 44 & $2 \cdot 175$ & 35 \\
\hline Siderite & $\mathrm{FeCO}_{3}$ & 0.810 & $1 \cdot 300$ & 36 & $2 \cdot 160$ & 35 \\
\hline Pyrite & $\mathrm{FeS}_{2}$ & 0.240 & $1 \cdot 230$ & 44 & 0.980 & 37 \\
\hline Haematite & $\mathrm{Fe}_{2} \mathrm{O}_{3}$ & $0 \cdot 190$ & 0.620 & 39 & $2 \cdot 175$ & 31 \\
\hline Olivine & $(\mathrm{Mg}, \mathrm{Fe})_{2} \mathrm{SiO}_{4}$ & $0 \cdot 150$ & $1 \cdot 275$ & 42 & 1.66 & 36 \\
\hline Glauconite & $\mathrm{K}_{2}(\mathrm{Mg}, \mathrm{Fe})_{2} \mathrm{Al}_{6}\left(\mathrm{Si}_{4} \mathrm{O}_{10}\right)_{3}(\mathrm{OH})_{12}$ & 0 & $0 \cdot 810$ & 43 & 0.420 & 36 \\
\hline $\mathrm{Fe}_{3} \mathrm{O}_{4}$ & $\mathrm{Fe}_{3} \mathrm{O}_{4}$ & 0 & 0.765 & 39 & $2 \cdot 185$ & 34 \\
\hline
\end{tabular}

When A. vinelandii strain UW was grown in the presence of natural iron-containing minerals, the limited amounts of available iron caused hyperproduction or repression of part or all of the siderophore system (Page \& Huyer, 1984; Table 1). The minerals used fell into three major classes: those which either enhanced the production of both azotobactin and catechols, enhanced the production of catechols only, or repressed azotobactin but allowed catechol production (Table 1). In each of these three cases the EA-insoluble catechol accounted for a constant 36 to $44 \%$ of the total catechol content. Similarly, strain UA1 hyperproduced catechols in response to the presence of the minerals and the EA-insoluble catechol remained at a constant 30 to $37 \%$ of the total catechol content (Table 1).

Incubation of strains UW or UAl in iron-limited medium for up to $36 \mathrm{~h}$, with or without added minerals, did not alter the percent of EA-insoluble catechol in the culture. Therefore, there was no simple conversion of azotochelin to the EA-insoluble catechol or vice versa in response to iron-starvation or after the use of the siderophores during iron transport. The EAinsoluble catechol also has been observed in iron-limited cultures of $A$. vinelandii ATCC 12837, 13705 and 12518 . The production of only azotochelin or only the EA-insoluble catechol has not yet been observed with any strain or cultural condition.

\section{Purification of the EA-insoluble catechol}

Batch treatment of iron-limited culture supernatant fluid with DEAE Sephadex A25 indicated that 2,3-DHBA and azotochelin were firmly bound, but the EA-insoluble catechol was easily removed by washing with aqueous $\mathrm{NaCl}$. This observation indicated that the EAinsoluble catechol was not simply an artefact created by the acidification of the culture fluid before EA extraction.

Large scale fractionation of the iron-limited culture supernatant fluid, however, usually involved the EA extraction step, to avoid permanent contamination of the column material. The EA-insoluble catechol was eluted from the DEAE Sephadex A25 column as a sharp peak with the initial distilled water wash, just before the start of the $\mathrm{NaCl}$ gradient (data not shown). The azotobactin also present in the aqueous phase was eluted with the $\mathrm{NaCl}$; further characterization of these fractions (W. J. Page, S. K. Collinson \& M. A. Abdallah) will appear elsewhere. The yield of $A_{310}$ material after this step was $60 \%$ of the starting material.

The fractions containing the EA-insoluble catechol were pooled, applied to a CM Sephadex column and eluted with an ammonium acetate gradient. Minor coloured impurities in the sample were removed by this step and the $A_{310}$ material eluted as a sharp peak at approximately $0.8 \mathrm{M}$-ammonium acetate (data not shown). The $A_{310}$ material in this peak accounted for $80 \%$ of 
the material applied to the column. The EA-insoluble catechol was found to be soluble in butanol, so extraction into butanol was used as a final purification step.

The spectrum of an aqueous solution of the purified EA-insoluble catechol had a maximum at $310 \mathrm{~nm}$, minimum at $275 \mathrm{~nm}$ and a maximum of $265 \mathrm{~nm}$, with an $A_{265}: A_{310}$ ratio of $2 \cdot 7$. Acidification resulted in a slight variation in this spectrum with a clear minimum developing at $235 \mathrm{~nm}$. Basic $\mathrm{pH}$ adjustment resulted in a complex spectrum having a strong absorption in the range 280 to $360 \mathrm{~nm}$ and a visible yellow colouration, characteristic of 2,3-DHBA at alkaline $\mathrm{pH}$ (Pittard et al., 1961). Acidification of an ethanolic solution of the EA-insoluble catechol did not result in an increased absorbance at $280 \mathrm{~nm}$, which suggested that an oxazoline ring was not present (Ong et al., 1979; Griffiths et al., 1984).

\section{Properties of the EA-insoluble catechol}

TLC in solvent system (A), used previously to resolve $A$. vinelandii catechols (Knosp et al., 1984), separated 2,3-DHBA from azotochelin but the EA-insoluble catechol did not move from the origin. In solvent system (B), the EA-insoluble catechol moved as a single spot $\left(R_{F} 0.42\right.$ to $0.47)$ with white-blue fluorescence under $366 \mathrm{~nm}$ light. The EA-insoluble catechol was also strongly reactive with ninhydrin, which indicated the presence of a primary amine. Therefore, the EA-insoluble catechol, presumed to be a siderophore of $A$. vinelandii, was named aminochelin.

\section{Stability and hydrolysis of aminochelin}

Aminochelin was notably unstable at alkaline $\mathrm{pH}$ at $20^{\circ} \mathrm{C}$ in the light. This was evident after $4 \mathrm{~h}$ in alkaline solution ( $5 \mathrm{mM}-\mathrm{NaOH}, \mathrm{pH} 10.5$ ) but was also noticeable in buffer at $\mathrm{pH} 7.5$ and to a lesser extent in buffer at $\mathrm{pH} 7 \cdot 0$, after $20 \mathrm{~h}$. The instability in these solutions was dimished at $4{ }^{\circ} \mathrm{C}$ but the trend towards a loss of the $A_{310}$ peak and development of a strong yellow colouration was apparent. Only solutions stored in distilled water, $\mathrm{pH} 5 \cdot 2$, or ammonium acetate buffer, $\mathrm{pH} 5.5$, in the dark at $4{ }^{\circ} \mathrm{C}$, or in $5 \mathrm{mM}-\mathrm{HCl}$, pH 2.5 , were stable.

When aminochelin that had been held in $\mathrm{NaOH}$ overnight at $20^{\circ} \mathrm{C}$ in the light was subjected to TLC in solvent system (B), the $R_{F} 0.47$ spot disappeared and was replaced by material which remained near the origin. Native aminochelin moved as a streak upon TLC in the alkaline solvent system (C), while $\mathrm{NaOH}$-treated aminochelin produced a fluorescent spot just off the origin and a ninhydrin-positive spot at $R_{F} \sim 0 \cdot 2$. Less severe alkaline treatment of aminochelin resulted in less intense spots and more streaking in solvent system $(C)$. These results suggested that alkaline treatment resulted in hydrolysis of aminochelin.

Acid hydrolysis of aminochelin resulted in the disappearance of the $R_{F} 0.47$ spot in solvent system (B) and the appearance of a fluorescent spot $\left(R_{F} 0 \cdot 70\right)$, a weakly ninhydrin-positive, weakly fluorescent, yellowish spot $\left(R_{F} 0.43\right)$, and a strong ninhydrin-positive spot $\left(R_{F} 0.15\right)$. The spectrum of the $R_{F} 0.43$ material resembled that of unhydrolysed aminochelin. Extraction of the hydrolysate with ether and TLC of the extract revealed that the $R_{F} 0.70$ spot was 2,3-DHBA.

The ninhydrin-positive spot was not lysine, as shown by TLC in solvent system (C). In this solvent, lysine moved off the origin $(0.7 \mathrm{~cm})$ while the hydrolysis product remained at the origin. TLC in solvent system (D) confirmed that the hydrolysis product $\left(R_{F} 0.08\right)$ was not asparagine, ornithine, arginine or lysine ( $R_{F} 0.20$ to 0.24$)$. The hydrolysis product did not correspond to a common amino acid, and notably threonine, which forms an oxazoline ring in several siderophores (Neilands, 1981), was absent. The low mobility of the hydrolysis product in solvent (D) indicated that it was more basic than lysine. Accordingly, the amino compound in the hydrolysate was found to have a mobility that corresponded to putrescine but not spermidine or spermine.

The mass of aminochelin was determined to be 225 , which corresponded to the protonated form of 2,3-dihydroxybenzoylputrescine. Sodium was present in the sample as evidenced by its association with the glycerol carrier. Similarly the ether-extracted hydrolysate contained a small amount of unhydrolysed material and masses at 110 and 131, which corresponded to the monoand di-sodium forms of putrescine. 
Table 2. ${ }^{55} \mathrm{Fe}$-uptake promoted by aminochelin and other siderophores produced by A. vinelandii

\begin{abstract}
Iron transport activity was assayed using $16 \mathrm{~h}$ iron-limited cells suspended in iron-limited Burk medium containing $10 \mathrm{mM}$-sodium citrate (Knosp et al., 1984) plus siderophore solutions with the absorbance characteristics indicated, in duplicate $10 \mathrm{~min}$ assays. Values for ${ }^{55} \mathrm{Fe}$ uptake have been corrected for ${ }^{55} \mathrm{Fe}$ bound to cells in the same solutions, but incubated on ice for $10 \mathrm{~min}$; reproducibility between duplicates was accurate to $\pm 5 \%$ of the mean.
\end{abstract}

\begin{tabular}{lllcc}
\multicolumn{1}{c}{ Siderophore } & $A_{410}$ & $A_{310}$ & $\begin{array}{c}{ }^{55} \text { Fe-uptake } \\
\text { (d.p.m.) }\end{array}$ & $\begin{array}{c}\text { Percentage of } \\
\text { maximum }\end{array}$ \\
Culture supernate & $0 \cdot 770$ & $0 \cdot 345$ & 43390 & ${ }^{55}$ Fe-uptake \\
& $0 \cdot 036$ & $0 \cdot 150$ & 47980 & 90 \\
Azotobactin & $0 \cdot 227$ & 0 & 39660 & 100 \\
& $0 \cdot 082$ & 0 & 46050 & 83 \\
Azotochelin & 0 & $0 \cdot 215$ & 21510 & 96 \\
& 0 & $0 \cdot 080$ & 20500 & 45 \\
Aminochelin & 0 & $0 \cdot 210$ & 37780 & 73 \\
& 0 & $0 \cdot 083$ & 31240 & 65 \\
Buffer only & 0 & 0 & 8680 & 18
\end{tabular}

Iron uptake promoted by aminochelin

Aminochelin $\left(A_{310}=0.3\right)$ formed a blue-purple coloured complex within $30 \mathrm{~s}$ with either $5 \times 10^{-5} \mathrm{M}-\mathrm{Fe}^{2+}\left(A_{\text {max. }} 500 \mathrm{~nm}\right)$ or $\mathrm{Fe}^{3+}\left(A_{\text {max. }} 485 \mathrm{~nm}\right)$ in distilled water, iron-limited Burk medium, or iron-limited Burk medium containing $10 \mathrm{~mm}$-sodium citrate, the latter to be used in ${ }^{55} \mathrm{Fe}$-uptake assays. Addition of $0.4 \mathrm{~mm}$-dithiothreitol to prevent oxidation of the $\mathrm{Fe}^{2+}$ ion did not alter this result. Aminochelin also reacted within $30 \mathrm{~s}$ with molybdate $\left(5 \times 10^{-5} \mathrm{M}\right)$, forming a yellow complex.

Aminochelin was an effective siderophore in iron-limited A. vinelandii, although the majority of the ${ }^{55} \mathrm{Fe}$-uptake in the unfractionated culture supernatant fluid was accounted for by azotobactin (Table 2). Maximum ${ }^{55} \mathrm{Fe}$-uptake by azotobactin was observed when the purified siderophore was diluted, possibly because salts that were inhibitory to siderophore-mediated transport (Knosp et al., 1984) were in the purified preparation. Azotochelin-mediated (Knosp et al., 1984) and aminochelin-mediated ${ }^{55} \mathrm{Fe}$-uptake were also salt sensitive; the latter was decreased $55 \%$ by the addition of $40 \mathrm{~mm}-\mathrm{NaCl}$ to the uptake medium (data not shown).

\title{
DISCUSSION
}

A. vinelandii produces three siderophores in response to iron-limitation. These include the yellow-green fluorescent compound azotobactin, the catechol azotochelin and the newly discovered EA-insoluble catechol which we have named aminochelin. Aminochelin has a simple structure which appears to be 2,3-dihydroxybenzoylputrescine (Fig. 3). This is the first report of a siderophore of this composition and containing this polyamine. As would be expected, the free amine of aminochelin makes it more hydrophilic than azotochelin and it is not extracted into EA. This property also distinguishes aminochelin from other catechol siderophores containing polyamines. For example, Paracoccus denitrificans produces $N^{1}, N^{8}$ bis(2,3-dihydroxybenzoyl)spermidine (compound II), an EA-soluble siderophore lacking a primary amine (Trait, 1975). The EA-soluble siderophores parabactin and agrobactin also contain compound II but are acylated at the secondary amide- $\mathrm{N}$ with a residue of salicylate or 2,3-DHBA, respectively, via an oxazoline ring (Neilands, 1981). Similarly, vibriobactin is a tricatechol with two oxazoline rings and a norspermidine backbone (Griffiths et al., 1984). These latter three siderophores have high affinities and specificity for $\mathrm{Fe}^{3+}$, a characteristic that was not found for aminochelin nor would it be expected from its simple structure.

Although aminochelin promotes ${ }^{55} \mathrm{Fe}$-uptake into $A$. vinelandii, it appears to be less efficient than azotochelin. The amount of ${ }^{55} \mathrm{Fe}$ transported by the two siderophores was $1.8 \times 10^{5}$ d.p.m. 


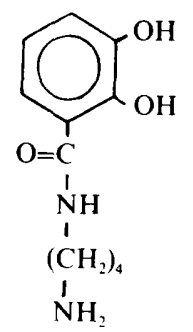

Fig. 3. Proposed aminochelin structure (2,3-dihydroxybenzoylputrescine).

$\left(A_{310} \text { aminochelin }\right)^{-1}$ versus $1.0 \times 10^{5}$ d.p.m. $\left(A_{310} \text { azotochelin }\right)^{-1}$ (Table 2$)$. Considering that azotochelin is a dicatechol, the amount of ${ }^{55} \mathrm{Fe}$ transported per mol of each siderophore would be approximately equal. However, a $2 \cdot 7$-fold dilution of the siderophores resulted in a $2 \%$ decline in azotochelin-mediated transport versus a $14 \%$ decline in aminochelin-mediated transport. Therefore, relatively high levels of aminochelin appear to be required to promote its maximal level of iron uptake.

Aminochelin is very stable at acidic $\mathrm{pH}$ and is increasingly less stable as the $\mathrm{pH}$ increases. Even at $\mathrm{pH} 7.0$ there is evidence of breakdown. This raises questions about its role in the alkaline environments preferred by $A$. vinelandii (Thompson \& Skerman, 1979). This siderophore and the others, however, are produced in vast excess of that required to promote rapid iron uptake, so continuous destruction of the siderophore may be tolerated provided an amount of active material remains.

One also must wonder why $A$. vinelandii produces three siderophores, two of which are not specific for $\mathrm{Fe}^{3+}$ and are incapable of high affinity binding of $\mathrm{Fe}^{3+} . A$. vinelandii has been isolated from mineral and calcareous soils (Thompson \& Skerman, 1979; Rennie, 1980) where the ability to solubilize mineral forms of iron, and perhaps other ions, would be advantageous. Catechols are effective agents in the solubilization of these minerals (this study; Page \& Huyer, 1984; Page \& von Tigerstrom, 1982) and it may be desirable not to have this nutritional system tightly controlled by iron. Aminochelin and azotochelin are produced coordinately, along with major iron-repressible outer membrane proteins of molecular mass 93000,85000 and 81000 , followed by azotobactin and a molecular mass 77000 iron-repressible outer membrane protein (Page \& Huyer, 1984). This suggests that there are two regulons affected by iron availability in $A$. vinelandii. The azotobactin regulon is most sensitive to iron availability, whereas the catechol regulon is expressed under conditions of iron repletion that are repressive to the azotobactin regulon. In $E$. coli, siderophore expression is negatively regulated by the Fur protein (Hantke, 1981, 1984). The Fur protein is activated by iron, which acts as a corepressor, promoting the binding of the Fur: Fe complex to the promotor regions of iron-regulated genes (De Lorenzo et $a l ., 1987)$. If a parallel system of regulation exists in $A$. vinelandii, differences between the promotor regions of the catechol and azotobactin regulons may similarly provide a basis for differential control by a single repressor complex. This possibility is presently under investigation.

We thank the Department of Chemistry, University of Alberta, for providing the mass spectroscopy data. This study was supported by a grant from the Natural Sciences and Engineering Research Council of Canada.

\section{REFERENCES}

ARNOw, L. E. (1937). Colorimetric determination of the components of 3,4-dihydroxyphenylalaninetyrosine mixtures. Journal of Biological Chemistry 118, 531-537.

Collinson, S. K., Doran, J. L. \& Page, W. J. (1987). Production of 3,4-dihydroxybenzoic acid by $\mathrm{Azo}$ monas macrocytogenes and Azotobacter paspali. Canadian Journal of Microbiology 33, 169-175.
Corbin, J. L. \& Bulen, W. A. (1969). The isolation and identification of 2,3-dihydroxybenzoic acid and 2- $N, 6-N$-di-(2,3-dihydroxybenzoyl)-L-lysine formed by iron-deficient Azotobacter vinelandii. Biochemistry 8, 757-762.

CSAKY, T. Z. (1948). On the estimation of bound hydroxylamine in biological materials. Acta chemica scandinavica 2, $450-454$. 
De Lorenzo, V., Wee, S., Herrero, M. \& Neilands, J. B. (1987). Operator sequences of the aerobactin operon of plasmid ColV-K30 binding the ferric uptake regulation ( fur) repressor. Journal of Bacteriology 169, 2624-2630.

Griffiths, G. L., Sigel, S. P., Payne, S. M. \& NeILANDS, J. B. (1984). Vibriobactin, a siderophore from Vibrio cholerae. Journal of Biological Chemistry 259, 383-385.

HANTKE, K. (1981). Regulation of ferric iron transport in Escherichia coli $\mathrm{K}-12$ : isolation of a constitutive mutant. Molecular and General Genetics 182, 288292.

HANTKE, K. (1984). Cloning of the repressor protein of iron-regulated systems in Escherichia coli K-12. Molecular and General Genetics 197, 337-341.

Knosp, O., von Tigerstrom, M. \& PAge, W. J. (1984). Siderophore-mediated uptake of iron in Azotobacter vinelandii. Journal of Bacteriology 159, 341-347.

Krebs, K. G., Heusser, D. \& Wimmer, H. (1969). Spray reagents. In Thin Layer Chromatography, pp. 854-909. Edited by E. Stahl. New York: SpringerVerlag.

NeIlands, J. B. (1981). Microbial iron compounds. Annual Review of Biochemistry 50, 715-731.

Ong, S. A., Peterson, T. \& Neilands, J. B. (1979). Agrobactin, a siderophore from Agrobacterium tumefaciens. Journal of Biological Chemistry 254, 1860 1865.

PAGE, W. J. (1987). Iron-dependent production of hydroxamate by sodium-dependent Azotobacter chroococcum. Applied and Environmental Microbiology 53, 1418-1424.

Page, W. J. \& Grant, G. A. (1987). Effect of mineral iron on the development of transformation compe- tence in Azotobacter vinelandii. FEMS Microbiology Letters 41, 257-261.

PAGE, W. J. \& HuYER, M. (1984). Derepression of the Azotobacter vinelandii siderophore system, using iron containing minerals to limit iron repletion. Journal of Bacteriology 158, 496-501.

PAGE, W. J. \& voN Tigerstrom, M. (1982). Iron- and molybdenum-repressible outer membrane proteins in competent Azotobacter vinelandii. Journal of Bacteriology 151, 237-242.

Pittard, A. J., Gibson, F. \& Doy, C. H. (1961). Phenolic compounds accumulated by washed cell suspensions of a tryptophan auxotroph of Aerobacter aerogenes. Biochimica et biophysica acta 49, 485494.

Post, E., Kleiner, D. \& Oelze, J. (1983). Whole cell respiration and nitrogenase activities in Azotobacter vinelandii growing in oxygen controlled continuous culture. Archives of Microbiology 134, 68-72.

RENNIE, R. J. (1980). Dinitrogen-fixing bacteria: computer-assisted identification of soil isolates. Canadian Journal of Microbiology 26, 1275-1283.

TAIT, G. T. (1975). The identification and biosynthesis of siderochromes formed by Micrococcus denitrifcans. Biochemical Journal 146, 191-204.

Thompson, J. P. \& Skerman, V. B. D. (1979). Azotobacteriaceae: the Taxonomy and Ecology of the Aerobic Nitrogen-fixing Bacteria. New York: Academic Press.

Westerveld, P., Bloom, M. L., Mabbott, G. A. \& FeKeTE, F. A. (1985). The isolation and identification of 3,4-dihydroxybenzoic acid formed by nitrogen-fixing Azomonas macrocytogenes. FEMS Microbiology Letters 30, 331-335. 